\title{
NRQCD, Effective Field Theories and Potential Models
}

\author{
Antonio Pineda ${ }^{\text {a* }}$ \\ aTheory Division, CERN, 1211 Geneva 23, Switzerland
}

\begin{abstract}
We review some recent developments on non-relativistic effective field theories for heavy quark-antiquark systems and how they can bring a well founded connection between QCD and potential models.
\end{abstract}

The study of the heavy quark-antiquark system is an old topic (see [i] for earlier references). Here we will concentrate on recent developments based on effective field theories. For large enough masses, these systems can be considered to be non-relativistic (NR) and are then characterized by, at least, three widely separated scales: hard (the mass $m$, of the heavy quarks), soft (the relative momentum of the heavy-quark-antiquark $|\mathbf{p}| \sim m v, v \ll 1$ ), and ultrasoft (US, the typical kinetic energy $E \sim m v^{2}$ of the heavy quark in the bound state system). In 1986, NRQED [2], an effective field theory for non-relativistic leptons, was presented, providing the first and decisive link in a chain of developments that is still growing. NRQED is obtained from QED by integrating out the hard scale $m$ (see [3] for some applications). It is characterized by an ultraviolet cut-off much smaller than the mass $m$ and much larger than any other scale. NRQCD [ 国 was born soon afterwards. NRQCD has proved to be extremely successful in studying $Q-\bar{Q}$ systems near threshold. The Lagrangian of NRQCD can be organized in powers of $1 / m$, thus making explicit the non-relativistic nature of the physical systems.

In order for effective field theories to be useful, a power counting is needed. The first power counting (organized in powers of $v$ and $\alpha_{s}$ ) used to assess the relative importance of the NRQCD matrix elements followed from arguments valid in the perturbative regime [5]. In particular, it was assumed that $\Lambda_{Q C D} \lesssim m v^{2}$. This implies that dynamics is mainly perturbative, with $v \sim$ $\alpha_{s}$, dictated by the solution of the Schrödinger

\footnotetext{
${ }^{*}$ Marie Curie Fellow, contract No. ERBFMBICT983405.
}

equation with the Coulomb potential $-C_{f} \alpha_{s} / r$. Therefore, it is debatable whether it could be applied for the charmonium system or for higher states of the bottomonium systems. Moreover, even in the perturbative case, because of the different scales involved in the problem, the matrix elements do not have a unique power counting but they also contribute to subleading orders in the $v$ counting, whereas the power counting in Ref. [5] only provided the leading order.

At this stage, there were two major questions (somewhat related) in the formulation of NRQCD: 1) the first was the non-existence of explicit power counting rules to systematically incorporate subleading effects, even in the perturbative situation; 2) the second was that the regularization procedure was based on cut-off regularization. Attempts to perform the matching between QCD and NRQCD using dimensional regularization had the drawback that the naive incorporation of the kinetic term in the quark propagator jeopardizes the power counting rules.

The solution to the latter problem was first given in Ref. 6]. There, it was argued that the matching between QCD and NRQCD in dimensional regularization should be performed just as in HQET -namely, the kinetic term must be treated as a perturbation and then the matching conditions and their Lagrangian in both theories are the same (in particular the matching computation in the effective theory is zero)- and performed for the terms bilinear in the fermions. For the four-fermion terms the matching along the same lines was worked out in Refs. [7],8]. The key point in the above derivations was that, in order to carry out the matching, it is not so important to know the power counting of each term in the 
effective theory as to know that the remaining dynamical scales of the effective theory are much lower than the mass. The power counting tells us the relative importance between different operators, but this does not change the value of the matching coefficients. That is, we only need

$$
m \gg|\mathbf{p}|, E, \Lambda_{Q C D} .
$$

As a matter of fact, this defines NRQCD in dimensional regularization (if it exists) irrespective of the relative size between $\Lambda_{Q C D}$ and the soft and ultrasoft scale, in particular, even if $\Lambda_{Q C D}$ were the next relevant scale.

The first problem, to obtain a complete power counting, was first studied within a mainly perturbative framework and triggered two lines of research:

A) On the one hand, by trying to classify the different momentum regions existing in a pure perturbative version of NRQCD and/or to reformulate NRQCD in ways where some of these regions were explicitly displayed by introducing new fields in the NRQCD Lagrangian. In particular, in Ref. [9], within a QED context, the existence of the ultrasoft region was first made explicit, besides the soft/potential one (of which the author only considered the leading order contribution somewhat missing the soft region as defined in [10]), and rules were given to estimate their size. Subsequent work 11] also tried to get complete power counting rules for NRQCD, but they also missed the soft region. Within this philosophy, in Ref. [10], the most complete classification of (perturbative) momentum regions to date was made by a rigorous diagrammatic study called the threshold expansion, including the soft region missed in these works. It was then realized that the hard region corresponded to the matching between NRQCD and QCD as described in Refs. [6, 8]. This allowed the computation of the electromagnetic current matching coefficient at two-loops [12]. Finally, some work was also done on formulating the threshold expansion of NRQCD within an effective Lagrangian formalism 13.

B) On the other hand, in parallel, a different approach was worked out in detail in [7, 14 to deal with the power-counting problem. It tried to an- swer the question: How would we like the effective theory for $\bar{Q}-Q$ systems near threshold to be? The first observation was that we did not want to describe all the degrees of freedom included in NRQCD, but rather only those with US energy. Therefore, the unwanted degrees of freedom should be integrated out. Moreover, we wanted to get a closer connection with a Schrödingerlike formulation for these systems (see also [15]). The idea was to connect NRQCD with potential models also, eventually, in the non-perturbative regime (more on that later). Roughly speaking we wanted our effective theory to be something like:

$$
\left.\begin{array}{l}
\left(i \partial_{0}-\frac{\mathbf{p}^{2}}{2 m}-V_{0}(r)\right) \Phi(\mathbf{r})=0 \\
\text { + corrections to the potential } \\
\text { +interaction with other low- } \\
\text { energy degrees of freedom }
\end{array}\right\} \text { pNRQCD }
$$

where $V_{0}(r)=-C_{f} \alpha_{s} / r$ in the perturbative case and $\Phi(\mathbf{r})$ is the $\bar{Q}-Q$ wave-function.

The resulting effective field theory was called potential NRQCD (pNRQCD). This is obtained after integrating out the soft scale, understood in the following way: pNRQCD is defined by two ultraviolet (UV) cut-offs $\Lambda_{1}$ and $\Lambda_{2}$. The former fulfils the relation $m v^{2} \ll \Lambda_{1} \ll m v$ and is the cut-off of the energy of the fermions and of the energy and the three-momentum of the gluons, whereas the latter fulfils $m v \ll \Lambda_{2} \ll m$ and is the cut-off of the relative three-momentum of the heavy fermion-antifermion system, p. This choice of the cutoffs can be motivated as follows. We are only interested in the degrees of freedom with US energy and, as a general philosophy, we would like to keep the UV cut-offs of the effective theory as low as possible. Therefore, any degree of freedom with larger energy should be integrated out. This fixes $\Lambda_{1}$ (we also fix the three-momentum cut-off of the gluons to be $\Lambda_{1}$ since the gluons satisfy a relativistic dispersion relation). The motivation for the choice of $\Lambda_{2}$ is that fermions with US energy have soft three-momentum owing to their non-relativistic dispersion relation. In short, we have only integrated out the degrees of freedom where a perturbative, order by order in $\alpha$, matching calculation 
can be performed (note that heavy fermions with soft three-momentum and US energy cannot be integrated out perturbatively since they are the responsible for producing the bound state). We believe that pNRQCD, defined in this way, has several advantges:

i) The matching between NRQCD and $\mathrm{pN}$ RQCD can be done order by order in $\alpha_{s}$ and in $1 / m$ (by analytically expanding the NRQCD Feynman diagrams in terms of the remaining dynamical scales of pNRQCD there is also an expansion in terms of the ultrasoft scale). In fact, this basically corresponds to obtaining the potential of the Schrödinger-like equation.

ii) The final effective theory resembles very much a Schrödinger-like equation. We believe that pNRQCD as described above provides a rigorous conection between NRQCD (quantum field theories) and potential models (non-relativistic quantum-mechanics formulation) in the situation where $\Lambda_{Q C D} \lesssim m v^{2}$.

iii) In spite of working with a Schrödinger-like equation, US gluons are still incorporated in a second-quantized, systematic and gauge-invariant fashion.

iv) The construction of pNRQCD follows a step-by-step procedure. It goes along the standard effective field theory idea of integrating out scale by scale 16], although properly speaking it should be said region by region. Since we are dealing with non-relativistic theories, the energy and the three-momentum are not related by the relativistic dispersion relation but by the nonrelativistic one, producing asymmetric ultraviolet cut-off in the effective theories. The advantage of working region by region is that in each (perturbative) region the Feynman integrals become homogeneous 2 , and much easier to compute in dimensional regularization, with just the one scale that we want to integrate out (on general grounds the matching calculation in each new effective theory is zero). On the other hand even in a non-perturbative situation it is expected that a better control in the dynamics would be obtained. In particular, perturbative regions can be disantangled from non-perturbative ones in a better

\footnotetext{
${ }^{2}$ I thank M. Beneke for stressing this point to me.
}

controlled way.

Finally we would like to note that, once the UV cut-offs of pNRQCD are fixed, no ambiguity is left in the definition of pNRQCD. Still, for an easy comparison with the first line of research mentioned above, we mention that pNRQCD is obtained from NRQCD after integrating out soft quarks and gluons and potential gluons. We would like to stress here that, in particular, the so-called soft region was already included in Ref. [7] that, as a matter of fact, was the first place where a power counting including the hard, soft, potential and ultrasoft region was given within an effective field theory framework.

Leaving aside non-perturbative effects, one could be worried about smaller dynamical scales $m v^{3}, \ldots$ that could appear by fine-tuning the energy to be near some poles. We would like to note that these eventual scales will not be a problem since they would be encoded in pNRQCD, but then it may be convenient to integrate out some further degrees of freedom to accurately describe physics at these smaller scales.

The developments in effective field theories explained above (with $\Lambda_{Q C D} \lesssim m v^{2}$ ) can be applied to several physical situations such as the $\Upsilon(1 S)$ 17], bottomonium sum rules 18 or $t-\bar{t}$ production near threshold [19], where we believe that put on more solid theoretical grounds the formalism used.

There has been some recent attempts to obtain renormalization group equations for nonrelativistic systems. For lack of space we do not discuss them here in detail (see 20]). We only briefly comment on the work of [21], from which it could be naively concluded that pNRQCD could not reproduce the anomalous dimension of the electromagnetic current. This is not true, which should be evident from the fact that the evaluation of the anomalous dimension in pNRQCD would go along similar lines to the one performed in [18. We believe that the approach in 21] may suffer from the drawback of having to treat the different scales entangled. This is in contradiction with the philosophy advocated here of working region by region and may jeopardize the huge simplification obtained in perturbative calculations. Moreover, since they treat the soft and US scale 
perturbatively, it is hard to imagine how to introduce non-perturbative effects in this approach.

So far we have restricted our considerations to the situation $\Lambda_{Q C D} \lesssim m v^{2}$. It is doubtful whether we can consider most of the charmonium and bottomonium spectrum to be in this situation but rather in the (generic) non-perturbative case with $m v \sim \Lambda_{Q C D}$. Then, it is not clear, a priori, what is the power counting that should be used for these systems 3. In particular, it is less clear how to obtain a rigorous connection between NRQCD and potential models (if it exists), although naively one would expect that, to some extent, the same philosophy as used previously to obtain pNRQCD could also be followed here. In this line of thinking, we would like to end by briefly reporting on some new results in our understanding of NRQCD and pNRQCD in the nonperturbative regime obtained in Ref. 23]; there, for the first time, a method to obtain a controlled derivation of the potential from QCD in terms of Wilson loops at arbitrary orders in $1 / m$ has been proposed. This has permitted to obtain new potentials for the heavy quarkonium (as well as for the heavy hybrids) at $O(1 / m)$ and $O\left(1 / m^{2}\right)$ previously missed in the literature, as well as to correct some of the already existing ones.

Acknowledgements. I thank J. Soto for his careful reading of the manuscript and M. Beneke for discussions.

\section{REFERENCES}

1. F.J. Ynduráin, "The Theory of Quarks and Gluon Interactions", third edition, Springer, Heidelberg, 1999.

2. W.E. Caswell and G.P. Lepage, Phys. Lett. B167, 437 (1986).

3. T. Kinoshita and M. Nio, Phys. Rev. D53, 4909 (1996); A.H. Hoang, P. Labelle and S.M. Zebarjad, Phys. Rev. Lett. 79, 3387 (1997); A. Czarnecki, K. Melnikov and A. Yelkhovsky, Phys. Rev. A59 4316 (1999).

\footnotetext{
${ }^{3}$ In fact, a different, non-standard, power counting of the matrix elements of NRQCD may eventually explain the apparent difficulties that NRQCD is facing to explain the polarization of prompt $J / \psi$ data, and to accurately determine the different matrix elements (see [22]).
}

4. B.A. Thacker and G.P. Lepage, Phys. Rev. D43, 196 (1991).

5. G.P. Lepage et al., Phys. Rev. D46, 4052 (1992).

6. A.V. Manohar, Phys. Rev. D56, 230 (1997).

7. A. Pineda and J. Soto, Nucl. Phys. B (Proc. Suppl.) 64, 428 (1998); Phys. Lett. B420, 391 (1998); Phys. Rev. D59, 016005 (1999).

8. A. Pineda and J. Soto, Phys. Rev. D58, 114011 (1998).

9. P. Labelle, Phys. Rev. D58, 093013 (1998).

10. M. Beneke and V.A. Smirnov, Nucl. Phys. B522, 321 (1998).

11. M. Luke and A.V. Manohar, Phys. Rev. D55, 4129 (1997); B. Grinstein and I.Z. Rothstein, Phys. Rev. D57, 78 (1998); M. Luke and M.J. Savage, Phys. Rev. D57, 413 (1998).

12. M. Beneke, A. Signer and V.A. Smirnov, Phys. Rev. Lett. 80, 2535 (1998); A. Czarnecki and K. Melnikov, Phys. Rev. Lett. 80, 2531 (1998).

13. H.W. Griesshammer, Phys. Rev. D 58, 094027 (1998)

14. N. Brambilla, A. Pineda, J. Soto and A. Vairo, Phys. Rev. D60, 091502 (1999); Nucl. Phys. B566, 275 (2000).

15. G.P. Lepage, nucl-th/9706029.

16. H. Georgi, Ann. Rev. Nucl. Part. Sci. 43, 209 (1993).

17. A. Pineda and F.J. Yndurain, Phys. Rev. D58, 094022 (1998).

18. K. Melnikov and A. Yelkhovsky, Phys. Rev. D59, 114009 (1999); A.A. Penin and A.A. Pivovarov, Phys. Lett. B435, 413 (1998); Nucl. Phys. B549, 217 (1999); A.H. Hoang, Phys. Rev. D61, 034005 (2000); M. Beneke and A. Signer, Phys. Lett. B471, 233 (1999).

19. A. H. Hoang et al., hep-ph/0001286.

20. A.V. Manohar, BEACH00 proceedings.

21. M.E. Luke, A.V. Manohar and I.Z. Rothstein, Phys. Rev. D61, 074025 (2000).

22. M. Kraemer, hep-ph/0010137; A.K. Leibovich, hep-ph/0008236.

23. N. Brambilla, A. Pineda, J. Soto and A. Vairo, hep-ph/0002250; A. Pineda and A. Vairo, hep-ph/0009145. 